\title{
Adenoviruses Type D in Patients with Respiratory Tract Infection Symptoms
}

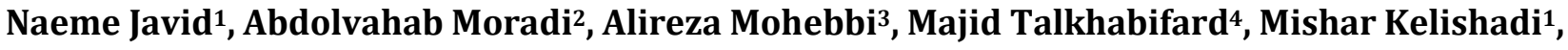 \\ Alijan Tabarraei ${ }^{*}$
}

\begin{abstract}
${ }^{1}$ Department of Microbiology, Infectious Diseases Research Centre, Golestan University of Medical Sciences, Gorgan, Iran ${ }^{2}$ Department of Microbiology, School of Medicine, Golestan University of Medical Sciences, Gorgan, Iran ${ }^{3}$ Student Research Committee, School of Medicine, Golestan University of Medical Sciences, Gorgan, Iran ${ }^{4}$ Department of Molecular Medicine, Faculty of Advanced Medical Sciences, Golestan University of Medical Sciences, Gorgan, Iran Email:naeme.javid@gmail.com, abmoradi@gmail.com, Mohebbi-a@goums.ac.ir, Majid.talkhabifard@gmail.com, kelishady@yahoo.com, *alijant@yahoo.com
\end{abstract}

How to cite this paper: Javid, N., Moradi, A., Mohebbi, A., Talkhabifard, M., Kelishadi, M. and Tabarraei, A. (2017) Adenoviruses Type D in Patients with Respiratory Tract Infection Symptoms. Open Access Library Journal, 4: e3624.

https://doi.org/10.4236/oalib.1103624

Received: April 20, 2017

Accepted: May 15, 2017

Published: May 18, 2017

Copyright $\odot 2017$ by authors and Open Access Library Inc.

This work is licensed under the Creative Commons Attribution International License (CC BY 4.0).

http://creativecommons.org/licenses/by/4.0/ c) (i) Open Access

\begin{abstract}
Different Human Adenoviruses (HAdV) serotypes are associated with epidemic respiratory infections. The aim of this study was to detect HAdV from patients with respiratory symptoms and typing virus circulating in North of IRAN. Four hundred nasopharyngeal aspirate samples were obtained from patients with respiratory symptoms. Clinical data were collected. After DNA extraction, Polymerase Chain Reaction (PCR) was done for conserved Hexon region. Positive PCR products were used for sequencing grouping. 37 cases (9.2\%) were positives. Clinical symptoms as cough (73\%), body $(67 / 6 \%)$, and fever $(64 / 9 \%)$ were found in 35 infected patients (94/5\%) and two patients (15/5\%) had no symptoms. All samples were belonged to HAdV of species D (HAdV-8). Prevalence of respiratory adenovirus infection in our area is consistent with other studies in Iran and ocular type of HAdV8 was the frequent adenovirus serotype circulating in acute respiratory disease in Golestan province.
\end{abstract}

\section{Subject Areas \\ Microbiology, Virology}

\section{Keywords}

Adenovirus Type D, Respiratory Tract Infection, HAdV 8

\section{Introduction}

Acute respiratory tract infection (ARTI) is one of the most common diseases in worldwide. World Health Organization (WHO) grades the lower RTIs as the main 
cause of disease, which is responsible for 4 million deaths per year. Different factors are involved including respiratory syncytial virus (RSV), influenza virus, parainfluenza virus (PIV), rhinovirus (RV), and human adenovirus (HAdV) [1].

HAdV is a member of the genus Mastadenovirus including seven A-G species. Sixty-eight types of Adenovirus have been reported. It has been revealed that species B (serotypes 3 and 7), C (serotypes 1, 2, and 5), and E (serotype 4) are generally associated with respiratory diseases, whereas Adenoviruses in species $\mathrm{F}$ and subspecies D are often associated in gastroenteritis and viruses keratoconjunctivitis, respectively [2].

Mild to acute respiratory illness in humans illustrated by Adenoviruses, as well as a diverse range of clinical symptoms based on the serotype, including gastroenteritis and ocular disease, with or without respiratory involvement. Different receptors dealing with interaction of host and viruses as species A, B, C, E, and F Adenoviruses utilize CD46, DSG-2, or CAR receptors and generally cause respiratory disease in humans, while species D Adenovirus bind to 22 -3-linked SA and GD1a glycans and has been associated with epidemic keratoconjunctivitis (EKC) which can progress to hemorrhagic conjunctivitis [3]. A recent study reported that species D can also be isolated in respiratory specimens as a dominant genotype [2].

However, human Adenovirus associated respiratory tract infection (RTI) is low. Respiratory viral infections represent the most common cause of acute illness. It accounts for $4 \%-10 \%$ cases of pneumonia, $2 \%-10 \%$ cases of bronchiolitis, and 3\% - 9\% cases of croup. Adenovirus infections can occur as epidemic, endemic or sporadic disease. This infection in children is ranged 2\% to $14 \%$ [4].

Association of respiratory viruses with human ocular disease are discussed and in vitro and in vivo study about ability of respiratory viruses to use the eye as a portal of entry and primary site of virus replication is highlighted and provides a needed bridge between clinical and laboratory studies of virus tropism [3].

AdVs serotype D (AdV-D), has shown to cause, in addition to ocular infection, respiratory illness [5]. Nasolacrimal system and receptor-mediated routes both are reported to be involved in AdVs-D respiratory infection. Moreover, cornea, conjunctiva, and nasolacrimal tissues are comprised of $\alpha 2$-3-linked sialic acids (SA) which are similar to those of the trachea, alveolus, bronchus, and bronchiole tissues in abdominal tract [6]. Therefore, it is thought that respiratory viral pathogens are able to infect the ocular system, vice versa. Control and treatment of disease presenting with ocular complications by Adenoviruses, understanding of the relationship between the development of ocular symptoms and respiratory disease is critical. Today has been growing interest in molecular typing adenovirus because clearly those specific serotypes are associated with disease severity [7]. Studying these pathogens in a larger context will be a good approach to understand the public health threat posed by these viruses and how best to prevent and treat complications.

The principal objectives of the present paper were to detect HAdV in NPA specimens from patients with respiratory symptoms and to determine the HAdV types that are circulating in Golestan Province, in North of IRAN. 


\section{Materials and Methods}

\subsection{Clinical Specimen}

Clinical samples were part of the sample collection of Reference Center for Influenza (Golestan University of medical science). 400 nasopharyngeal aspirate samples were collected between November 2010 and April 2012 from patients with respiratory symptoms during influenza pandemic. Nasopharyngeal specimens were taken and transferred to the virology laboratory in VTM transport medium (VTM consists of Modified Hank's Balanced Salt Solution supplemented with bovine serum albumin, sucrose, glutamic acid, gelatin, Phenol red and antibiotic with $\mathrm{pH} 7.3 \pm 0.2$ ). Demographic and clinical data were collected (Ethic No. 911103196).

\subsection{DNA Extraction}

Extraction of DNA from $200 \mu \mathrm{l}$ of clinical specimen performed by using the High Pure Viral Nucleic Acid Extraction Kit (Roche) according to the manufacturer's instruction.

\subsection{Polymerase Chain Reaction (PCR) Performance}

The partial hexon gene was amplified using the primer pair, ADHEXF 5'CAACACCTAYGASTACATGAA-3' and ADHEXR 5'-KATGG GGTAR AGCA TGTT3 ' To amplify a $475 \mathrm{bp}$ fragment of the partial hexon gene [8].

PCR: PCR was performed in $50 \mu$ volumes containing $1 \times$ PCR Buffer $(10 \mathrm{mM}$ Tris- $\mathrm{HCl}$ [pH 8.3], $50 \mathrm{mM} \mathrm{KCl),} 2 \mathrm{mM} \mathrm{MgCl} 2,200 \mathrm{mM}$ each deoxynucleoside triphosphate, 20 pmol of each primer, 2.5 Unit of Taq DNA polymerase [Genet Bio] and $5 \mu \mathrm{l}$ of DNA template. The reaction was carried out in a thermal cycler (Peqlab) with the following settings: initial denaturation for 5 minutes at $94^{\circ} \mathrm{C}$, followed by 35 cycles of denaturation at $94^{\circ} \mathrm{C}$ for $1 \mathrm{~min}$, annealing at $50^{\circ} \mathrm{C}$ for 1 min, and extension at $68^{\circ} \mathrm{C}$ for $1 \mathrm{~min}$. A final extension at $68^{\circ} \mathrm{C}$ for 5 min was also proposed. Standard precautions were taken to prevent PCR contamination. PCR products on $1.5 \%$ agarose gels stained by ethidium bromide and visualized under UV trans-illuminator (Figure 1).

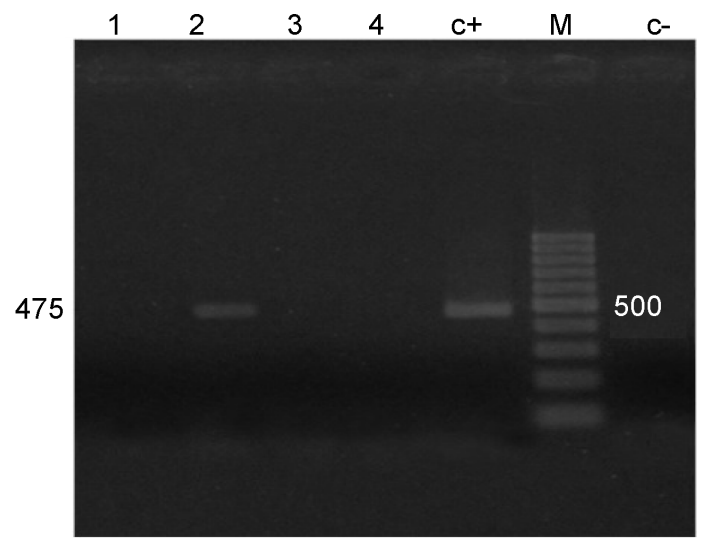

Figure 1. M: DNA molecular marker, $\mathrm{c}+$ : positive Control, c-: negative control and 2: positive clinical specimen. 
All positive PCR products purified and sequenced in both directions by Sanger methods (Macrogen, South Korea). PCR products were sequenced directly after purification.

\subsection{Data Analysis}

All data analyzed by SPSS-16 using T-test and Chi-square analytical tests. Pvalue of less than 0.05 was considered as statistically significant. The sequence data were analyzed using Sequencer software. All sequences were imported to the BLAST (http://blast.ncbi.nlm.nih.gov/Blast.cgi) and aligned against the GenBank database. Sequence alignments were built with BioEdit alignment editor software (version 5.0.9; Tom Hall, North Carolina State University) and a phylogenetic dendrogram was constructed using the neighbor-joining method with the MEGA program (Sudhir Kumar, Arizona State University); and the validity of the dendrogram was estimated with 100 bootstrap pseudo-replicates.

\section{Result}

\subsection{Demographic and Clinical Characteristics of Patients}

400 nasopharyngeal swabs were processed, 55 (13.8\%), 316 (79\%) and 29 (7.2\%) samples were belonged to the patients younger than 20 , between $20-40$, and older than 40 years old, respectively. 31 (7.8\%) of samples were males and 369 (92.2\%) were females of which, 369 women, 121 (32.8\%) cases were pregnant. ARTI symptoms associated with fever, cough, and muscle ache were seen in 247 (61.7\%), 299 (74.7\%), and 254 (63.5\%) patients, respectively. 330 (82.5\%) of patients had one symptom at least.

Moreover, 37 cases $(9.2 \%)$ were infected with adenovirus. In those, clinical symptoms were as follow, cough in 27 (73\%), body pain in $25(67 / 6 \%)$, and fever in 24 cases $(64 / 9 \%)$ were found as the most common clinical signs and 35 patients $(94 / 5 \%)$ had at least one symptom.

Seasonal distribution pattern of positive cases was showed that 26 cases were belonging to cold season and 11 cases collected from warm season. There was no significant relationship between positive sample and season. None of the patients was undergone antiviral treatment and no significant correlation was found between age, sex, season and positive cases.

Generally, there were no detectable significant differences in the clinical presentation as well as the gender and duration of illness in patients.

\subsection{PCR Specificity}

The identification of positive samples was confirmed by PCR and sequencing. In addition, specificity of primer was confirmed by PCR performance on other non-AdV related viruses.

\subsection{Sequencing and Phylogenetic Tree Results}

All of the positive samples were sequenced (Online Resource 1). Degenerated primers targeting the hexon gene (described Casas and et al.) were able to ampl- 
ify all of the HAdV reference strain.

Through a BLAST search with sequences obtained in this study, we found that all samples belonged to HAdV of species D (HAdV-8). One hundred percent of concurrence was acquired with existing GenBank sequences for serotypes 8 . Moreover, a phylogenetic tree was constructed (Figure 2) using the sequences obtained in this study and 3 different HAdV reference sequences obtained from GenBank (http://www.ncbi.nlm.nih.gov/Genbank), which corroborated the results obtained using BLAST.

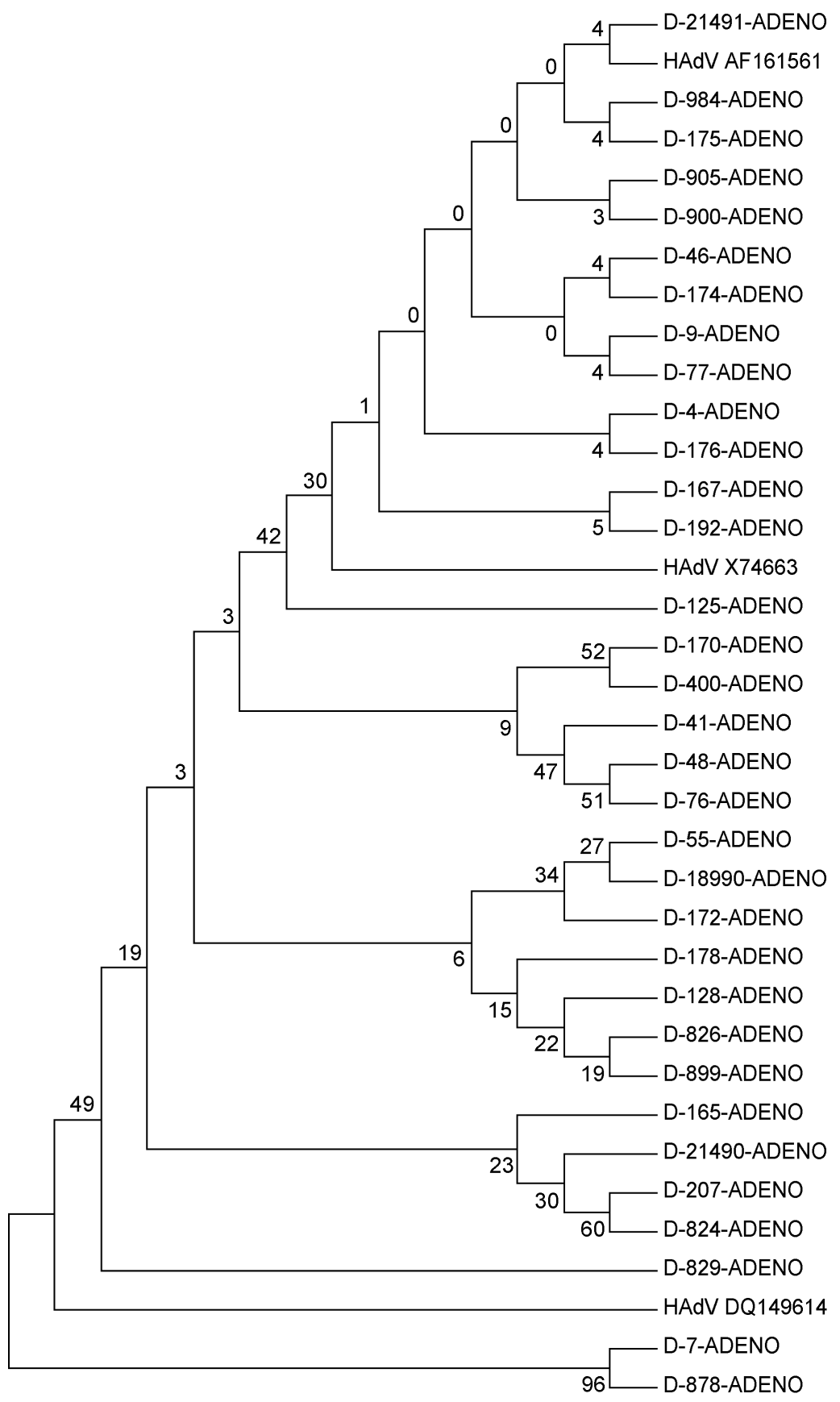

Figure 2. Phylogenetic tree created based on partial hexon gene and with 100 bootstrap pseudo-replicates. 


\section{Discussion}

Here, we have detected HAdV in Golestan province in North of Iran, where HAdV-D8 was identified as dominated HAdV type is described.

Genotype 8 of HadV-D is reported to be most prevalent in ocular complications through all season with no differences in susceptibility in male or female [2] [9]. Contrarily, most HadV associated respiratory illness has been in cold months [2]. It is also notable that ocular-related HAdV is more prevalent than young children [2] [10]. Here we have found a peak of infection in the cold season but it was not significant. Additionally, it was found that HAdV-Distribution is normal through either male or female, as well as age.

Human adenoviruses are important causes of respiratory tract infections and are responsible for one third of deaths in children and adults in worldwide [11].

The identification of HAdV serotypes usually based on cell culture method, which is in turn, relies on neutralization tests or hemagglutinin inhibition of the samples. Although, these methods are time-consuming and depend on enough products of infectious viruses and as a result, are not appropriate for the sensitive and fast identification of HAdV. Identification of HAdV in clinical sample by PCR method has become popular alternative methods that permit sensitive, fast, and accurate molecular detection [12] [13].

A PCR assay, enforceable to clinical sample, was developed that could detect all known serotypes of HAdV with high sensitivity and allow for serotype determination by sequencing of the amplicons. The primer pair selected is based on conserved sequences of the hexon gene [10].

There are few epidemiological studies of adenovirus infections in IRAN and no information is available on the distribution of serotypes. In total of 400 patients, 37 cases (9.2\%) were infected with adenovirus that the frequency was similar to study of Echavaria and his Colleagues in Argentina [14] and that is lower than the frequency of $14.4 \%$ and $22 \%$ reported by another study from Iran [1] [15] [16] and several parts of the world including Oman (15\%), Kenya (14\%), Korea (10.3\%), Canada (7.73\%), Brazil (7.1\%), Australia (7.3\%), Hung Kong (5.3\%), Mejia (5.2\%), and China (4.9\%) [1] [17] [18].

In a similar study, Adenovirus serotypes 7 and 3 were most commonly identified serotype in respiratory disease. Prevalent serotype in Brazil was Ad-4, and several parts of the world including Taiwan (Ad-3), USA (Ad-14, Ad-3 and Ad-7), and Canada (Ad-3, Ad-2, Ad-1 and Ad-21, respectively) [17] [18] [19] [20] [21].

Through a BLAST search with sequences obtained from all amplicons, we determined that all samples (100\%) belong to HAdV-8. Nevertheless, serotype 8 of adenovirus doesn't common serotype in respiratory infection.

As it has been known that HadV serotype D was associated with ocular diseases [6]. However, it is believed HadV serotype D is able to, according to same viral receptors in eyes or intestinal epithelium, cause infection [3]. In addition, there are other studies implicating ocular and respiratory tract infections with other viruses [22]. Therefore, respiratory viruses may infect intestinal epithe- 
liums following primarily ocular infection [3].

Also samples collection was done in pandemic influenza thus perhaps HAdV8 was common in during of pandemic influenza in this region.

This comprehensive means of typing adenovirus allows public health laboratories to inform their stakeholders on the possibility of new emergent strains, which in turn should allow stakeholders to undertake appropriate infection control and public health steps when these strains arrive in a region. It is evident from this study that sequence-based methodologies for the typing of adenovirus from viral culture materials are an effective means of determining adenovirus serotypes in specific communities and populations.

Epidemiological surveillance of HAdV serotypes will improve our understanding of the global burden of HAdV infection.

Molecular methods were found to be useful for rapid diagnosis of adenovirus infections and it will be important for control and treatment of infection. According to the results of the prevalence of respiratory adenovirus infection is consistent with other studies in Iran and other countries but obtained serotype is different in this region. This study indicates that HAdV8 was the most common adenovirus serotype circulating in acute respiratory disease in the south east of the Caspian Sea between November 2010 and April 2012.

\section{Acknowledgements}

This study was supported by Golestan research center of infectious disease and Golestan University of Medical sciences, Golestan, IRAN.

\section{Conflict of Interests}

The Authors declares that there is no conflict of interest.

\section{References}

[1] Moattari, A., Emami, A., Pirbonyeh, N. and Yaghoobi, R. (2014) Detection of Adenovirus Infection among Children with Acute Respiratory Disease during 20102012 in Shiraz, Iran. Archives of Pediatric Infectious Diseases, 2, e15930.

[2] Akhil, C., Suresha, P.G., Sabeena, S., Hindol, M. and Arunkumar, G. (2016) Genotyping of Human Adenoviruses Circulating in Southwest India. VirusDisease, 27, 266-270. https://doi.org/10.1007/s13337-016-0337-5

[3] Belser, J.A., Rota, P.A. and Tumpey, T.M. (2013) Ocular Tropism of Respiratory Viruses. Microbiology and Molecular Biology Reviews, 77, 144-156. https://doi.org/10.1128/MMBR.00058-12

[4] Hong, J.-Y., Lee, H.-J., Piedra, P.A., Choi, E.-H., Park, K.-H., Koh, Y.-Y., et al. (2001) Lower Respiratory Tract Infections Due to Adenovirus in Hospitalized Korean Children: Epidemiology, Clinical Features, and Prognosis. Clinical Infectious Diseases, 32, 1423-1429. https://doi.org/10.1086/320146

[5] Belsy, A., Odalys, V., Alexander, P., Clara, S., Angel, G., Grehete, G., et al. (2009) Molecular Characterization of Adenoviral Infections in Cuba: Report of an Unusual Association of Species D Adenoviruses with Different Clinical Syndromes. Archives of Virology, 154, 619-627. https://doi.org/10.1007/s00705-009-0338-4

[6] Jin, Y., Zhang, R.-F., Xie, Z.-P., Yan, K.-L., Gao, H.-C., Song, J.-R., et al. (2013) 
Prevalence of Adenovirus in Children with Acute Respiratory Tract Infection in Lanzhou, China. Virology Journal, 10, 271. https://doi.org/10.1186/1743-422X-10-271

[7] Xu, W., McDonough, M.C. and Erdman, D.D. (2000) Species-Specific Identification of Human Adenoviruses by a Multiplex PCR Assay. Journal of Clinical Microbiology, 38, 4114-4120.

[8] Casas, I., Avellon, A., Mosquera, M., Jabado, O., Echevarria, J., Campos, R., et al. (2005) Molecular Identification of Adenoviruses in Clinical Samples by Analyzing a Partial Hexon Genomic Region. Journal of Clinical Microbiology, 43, 6176-6182. https://doi.org/10.1128/JCM.43.12.6176-6182.2005

[9] Erdin, B.N., Pas, S.D., Durak, I., Schutten, M. and Sayıner, A.A. (2015) A 5-Year Study of Adenoviruses Causing Conjunctivitis in Izmir, Turkey. Journal of Medical Virology, 87, 472-477. https://doi.org/10.1002/jmv.24071

[10] Demian, P.N., Horton, K.C., Kajon, A., Siam, R., Hasanin, A.M.N., Sheta, A.E., et al. (2014) Molecular Identification of Adenoviruses Associated with Respiratory Infection in Egypt from 2003 to 2010. BMC Infectious Diseases, 14, 50. https://doi.org/10.1186/1471-2334-14-50

[11] Marinheiro, J.C., Sanalios, R.B., dos Santos, C., Alves da Costa, C. and Hársi, C.M. (2009) Duplex-PCR Assay for the Detection of Adenovirus and Respiratory Syncytial Virus in Nasopharyngeal Samples. Memórias do Instituto Oswaldo Cruz, 104, 118-120. https://doi.org/10.1590/S0074-02762009000100018

[12] Osiowy, C. (1998) Direct Detection of Respiratory Syncytial Virus, Parainfluenza Virus, and Adenovirus in Clinical Respiratory Specimens by A Multiplex Reverse Transcription-PCR Assay. Journal of Clinical Microbiology, 36, 3149-3154.

[13] Lee, J.A., Kim, N.H., Kim, S.J., Choi, E.H. and Lee, H.J. (2005) Rapid Identification of Human Adenovirus Types 3 and 7 from Respiratory Specimens via Multiplex Type-Specific PCR. Journal of Clinical Microbiology, 43, 5509-5514. https://doi.org/10.1128/JCM.43.11.5509-5514.2005

[14] Echavarria, M., Sanchez, J.L., Kolavic-Gray, S.A., Polyak, C.S., Mitchell-Raymundo, F., Innis, B.L., et al. (2003) Rapid Detection of Adenovirus in Throat Swab Specimens by PCR during Respiratory Disease Outbreaks among Military Recruits. Journal of Clinical Microbiology, 41, 810-812. https://doi.org/10.1128/JCM.41.2.810-812.2003

[15] Malekshahi, S.S., Azad, T.M., Yavarian, J., Shahmahmoodi, S., Naseri, M. and Rezaei, F. (2010) Molecular Detection of Respiratory Viruses in Clinical Specimens from Children with Acute Respiratory Disease in Iran. The Pediatric Infectious Disease Journal, 29, 931-933.

[16] Kahbazi, M., Fahmizad, A., Armin, S., Ghanaee, R., Fallah, F., Shiva, F., et al. (2011) Aetiology of Upper Respiratory Tract Infections in Children in Arak City: A Community Based Study. Acta Microbiologica et Immunologica Hungarica, 58, 289-296. https://doi.org/10.1556/AMicr.58.2011.4.5

[17] Luiz, L.N., Leite, J.P.G., Yokosawa, J., Carneiro, B.M., Pereira Filho, E., et al. (2010) Molecular Characterization of Adenoviruses from Children Presenting with Acute Respiratory Disease in Uberlândia, Minas Gerais, Brazil, and Detection of an Isolate Genetically Related to Feline Adenovirus. Memórias do Instituto Oswaldo Cruz, 105, 712-716. https://doi.org/10.1590/S0074-02762010000500019

[18] Yeung, R., Eshaghi, A., Lombos, E., Blair, J., Mazzulli, T., Burton, L., et al. (2009) Characterization of Culture-Positive Adenovirus Serotypes from Respiratory Specimens in Toronto, Ontario, Canada: September 2007-June 2008. Virology Journal, 6, 11. https://doi.org/10.1186/1743-422X-6-11 
[19] Ryan, M.A., Gray, G.C., Smith, B., McKeehan, J.A., Hawksworth, A.W. and Malasig, M.D. (2002) Large Epidemic of Respiratory Illness Due to Adenovirus Types 7 and 3 in Healthy Young Adults. Clinical Infectious Diseases, 34, 577-582. https://doi.org/10.1086/338471

[20] Hsieh, W.-Y., Chiu, N.-C., Chi, H., Huang, F.-Y. and Hung, C.-C. (2009) Respiratory Adenoviral Infections in Taiwanese Children: A Hospital-Based Study. Journal of Microbiology, Immunology, and Infection, 42, 371-377.

[21] Tate, J.E., Bunning, M.L., Lott, L., Lu, X., Su, J., Metzgar, D., et al. (2009) Outbreak of Severe Respiratory Disease Associated with Emergent Human Adenovirus Serotype 14 at a US Air Force Training Facility in 2007. Journal of Infectious Diseases, 199, 1419-1426. https://doi.org/10.1086/598520

[22] Hall, C.B., Douglas, R., Schnabel, K.C. and Geiman, J.M. (1981) Infectivity of Respiratory Syncytial Virus by Various Routes of Inoculation. Infection and Immunity, 33, 779-783.

Submit or recommend next manuscript to OALib Journal and we will provide best service for you:

- Publication frequency: Monthly

- 9 subject areas of science, technology and medicine

- Fair and rigorous peer-review system

- Fast publication process

- Article promotion in various social networking sites (LinkedIn, Facebook, Twitter, etc.)

- Maximum dissemination of your research work

Submit Your Paper Online: Click Here to Submit

Or Contact service@oalib.com 